\title{
Cervical smear screening in a tertiary care gynaecological clinic in Sri Lanka: A survey from a middle income country
}

\author{
Patabendige $\mathrm{M}^{1}$, Perera $\mathrm{SS}^{2}$, Wickramasooriya $\mathrm{DJ}^{3}$, Jayawardane $\mathrm{A}^{4}$ \\ ${ }^{1}$ Castle Street Hospital for Women, Colombo, Sri Lanka. \\ ${ }^{2}$ Department of Obstetrics and Gynaecology, Faculty of Medicine, University of Kelaniya, Ragama, Sri Lanka. \\ ${ }^{3}$ Department of Pathology, Faculty of Medicine, University of Ruhuna, Galle, Sri Lanka, \\ ${ }^{4}$ Department of Obstetrics and Gynaecology, Faculty of Medicine, University of Colombo, Sri Lanka.
}

Correspondence: Dr. Malitha Patabendige

e-mail: mpatabendige@gmail.com

(D) https://orcid.org/0000-0002-4092-7092

Submitted on 07.05.2021 and accepted for publication on 30.06.2021

\begin{abstract}
Introduction: Awareness and knowledge about cervical smear are lacking among the women in the developing world. The aim of the study was to study the awareness and knowledge regarding cervical smear screening among a cohort of Sri Lankan women.

Methods: A cross-sectional study was conducted at a gynaecology clinic in a tertiary care hospital, Sri Lanka from January to August 2018. A consecutively recruited clinic attendees who attended the clinic for the first-time; aged over 35 years were interviewed using a previously piloted questionnaire. Outcome measures were socio-demographic and clinical characteristics, knowledge about cervical smear screening and uptake of Pap smear testing.

Results: Among the 165 women interviewed, 146 (88.5\%) had heard about cervical carcinoma. Median (IQR) age was 46.0 (39.0-54.0) years. Only 75.2\% had heard about cervical screening. Knowledge about cervical smear screening was low and $60 \%$ of women were not worried about themselves getting cervical carcinoma. Awareness about cervical smear screening was $58.8 \%$ and only $78 / 165$ (47.3\%) had ever undergone screening. Fear of vaginal examination and lack of awareness was noted in $6.7 \%$ and $29.7 \%$ of them respectively. Public health midwives were the commonest source of information to them regarding cervical smear.
\end{abstract}

Conclusion: Awareness and knowledge regarding cervical carcinoma is poor in the study sample.

Keywords: Cervical cancer, Pap smear, cervical screening, uterine cervical diseases/ prevention and control.

\section{Introduction}

According to statistics of the national epidemiology unit, cervical cancers are the second most common female malignancies in Sri Lanka and accounts for $10 \%$ of all female malignancies $(1,2)$. Worldwide, approximately 500,000 new cases and 274,000 deaths are attributable to cervical malignancy annually, making cervical malignancy the second most common cause of death from malignancy in women in the world (3-5). The American College of Obstetricians \& Gynaecologists (ACOG) report that the incidence of cervical malignancy has decreased by more than $50 \%$ in the past $30+$ years, largely due to the increasing use of cervical malignancy screening with cervical cytology in 
USA(6). According to the WHO, worldwide cervical malignancy rates have decreased dramatically with the increase use of screening tests, however, incidence and prevalence in developing countries remains high due to lack of screening programmes, with approximately $80 \%$ of all cervical cancer deaths occurring in low- and middle- income countries (LMIC) (3). These data clearly illustrate that LMICs are in need of aggressive strategies to reduce the burden of cervical carcinoma $(5,7)$.

In Sri Lanka, each Medical Officer of Health area has a special clinic for the well-being of women over 35 years and these clinics are known as 'WellWoman Clinics' functioning all over the country. Cervical smear testing through Pap smear method is offered to females above 35 years of age free of charge and the test is repeated once in every five years when the cytology is reported normal (8). Despite the regional availability of aforementioned 'Well-woman Clinics' and gynaecological hospital clinics for screening, cervical malignancy remains the second most common malignancy among females in Sri Lanka (1).

It is important to assess the awareness and knowledge about cervical smear among the target population. Objectives of this study were to study awareness and knowledge regarding cervical smear screening with a view to find out any reasons for higher cervical malignancy rate despite having a screening programme.

\section{Methods}

A descriptive cross-sectional study was performed in four gynaecological clinics at University Gynaecology Unit, North Colombo Teaching Hospital (NCTH), Ragama, Sri Lanka, from January to August 2018. All the consecutive first visit women aged 35 years or above who were attending the university gynaecology clinic were selected by the consecutive sampling method. Women with follow up visits were excluded as clinic protocol was to offer opportunistic screening if a test was missed or due.

Eligible women were invited by investigators at each study setting. Before entry into the study, the aims, methods, reasonably anticipated benefits and potential hazards of the study were explained and informed consent obtained. Basic sociodemographic details, awareness of cervical smear screening including sources of information, knowledge regarding cervical smear screening (assessed by eight closed-ended questions) and details of previous cervical smear testing were collected using an interviewer-administered questionnaire which was piloted using a sample of 10 volunteer clinic attendees from a gynaecology clinic in another hospital.

Outcome measures were socio-demographic and clinical characteristics of women, awareness and knowledge about cervical smear screening and uptake of cervical smear testing. Descriptive statistics were used to summarize the data. Continuous variables were presented as mean (standard deviation) if they are normally distributed; otherwise as median (interquartile range). Logistic regression analysis was conducted to evaluate the association between awareness of cervical smear screening as the dependent variable with the age, parity, income, educational level as covariates. $p$ value $<0.05$ was considered as statistically significant. Ethical approval for the study was obtained from the Ethics Review Committee, Faculty of Medicine, University of Kelaniya, Ragama, Sri Lanka.

\section{Results}

A total of 165 women were studied and 146/165 $(88.5 \%)$ had heard about cervical carcinoma. Median (interquartile range) age was 48.4 (40) years. Basic demographic characteristics have been summarised in Table 1.

Only 124/165 (75.2\%) have heard about cervical screening. Table 2 shows the knowledge about cervical smear screening in response to the eight questions in the questionnaire. Twenty-five (15.2\%) subjects had personally known a person with cervical carcinoma. Ninety nine $(60.0 \%)$, were not much concerned about getting a cervical carcinoma for themselves. Only 97/165 (58.8\%) were aware of screening; 43/165 (26.1\%) have had their last cervical screening within the last five years. Moreover, 26/165 (15.8\%) were not aware about the well woman clinics in Sri Lanka and 60/165 (36.4\%) women were not aware about 
a health facility where cervical screening is being performed. Eighty-seven (52.7\%) have never undergone cervical smear screening. Out of subjects who have undergone the screening test, only 66 (84.6\%) have sought further medical advice regarding the result of the cervical smear test. Reason for not taking the cervical smear test are summerised in Table 3.
Table 4 shows the sources of information for cervical screening and this may yield positive insights in planning future strategies in LMIC towards improving cervical cancer screening. Logistic regression analysis was done assessing the awareness about cervical smear screening with demographic parameters including age $(p=0.84)$, parity $(p=0.15)$, income $(p=0.93)$, employment status $(p=0.99)$ and educational level $(p=0.008)$.

Table 1: Demographic characteristics of the participants

\begin{tabular}{|c|c|c|}
\hline Item & n (\%) & Median (IQR) ${ }^{\#}$ \\
\hline Age & - & $46.0(39.0-54.0)$ \\
\hline Marital status: ever-married ${ }^{*}$ & $164(99.4)$ & - \\
\hline Occupation & $51(30.9)$ & - \\
\hline Educational level & & - \\
\hline No formal education & $5 \quad(3)$ & - \\
\hline Primary education only & $34(20.6)$ & - \\
\hline Up to G.C.E. O/L & $92(55.8)$ & - \\
\hline Up to G.C.E. A/L & $28(17)$ & - \\
\hline Higher education & $6 \quad(3.6)$ & - \\
\hline Parity & - & $2.0(2.0-3.0)$ \\
\hline Age at first sexual intercourse & - & $23.0(19.5-27.0)$ \\
\hline Employed & $51(30.9)$ & - \\
\hline \multicolumn{3}{|l|}{ Monthly income (LKR) } \\
\hline$<20,000$ & $68(41.2)$ & - \\
\hline $20,000-40,000$ & $71(43)$ & - \\
\hline $40,000-60,000$ & $14 \quad(8.5)$ & - \\
\hline $60,000<$ & $12 \quad(7.3)$ & - \\
\hline \multicolumn{3}{|c|}{ Median (IQR) have been computed for the numerical data only ${ }^{\#}$} \\
\hline \multicolumn{3}{|c|}{ Ever-married women were those who have been married at least once in their lives" } \\
\hline $\begin{array}{l}\text { IQR: Interquartile range, G.C.E. O/L: Gen } \\
\text { of Education Advanced Level, LKR: Sri L }\end{array}$ & ducation Ordinary & E. A/L: General Certificate \\
\hline
\end{tabular}


Table 2: Factors related to the level of knowledge on Pap smear screening

\begin{tabular}{ll}
\hline Item $(\mathbf{N}=\mathbf{1 6 5})$ & n (\%) \\
\hline Knowledge on cervical cancer and Pap smear testing & $124(75.2)$ \\
1. Heard about Pap smear test & $97(58.8)$ \\
2. Pap smear test is done as a screening test for cervical cancer & $89(53.9)$ \\
3. Pap smear detects early cancers & $73(44.2)$ \\
4. The age at which Pap smear test needs to be started & $52(31.5)$ \\
5. The interval between Pap smear tests & $51(30.9)$ \\
6. Cervical cancer is acquired sexually & $46(27.9)$ \\
7. Cervical cancer is caused by a virus & $74(44.8)$ \\
8. Cervical cancer can be prevented &
\end{tabular}

Table 3: Reasons for not taking the Pap smear test

\begin{tabular}{lcc}
\hline Reason for not taking the Pap smear test $(\mathbf{N}=\mathbf{1 6 5})$ & n (\%) \\
\hline Fear of vaginal examination/ embarrassment & 11 & $(6.7)$ \\
Lack of any symptoms & 24 & $(14.6)$ \\
Unaware about it & 49 & $(29.7)$ \\
Never requested by a doctor & 5 & $(3.0)$ \\
Negligence & 13 & $(7.9)$ \\
Feeling that it is not important & 3 & $(1.8)$ \\
Missing data & 60 & $(36.4)$ \\
\hline
\end{tabular}

Table 4: Source of information regarding Pap smear screening

\begin{tabular}{lc}
\hline Source of information of Pap smear screening $(\mathbf{N}=\mathbf{1 6 5})$ & Frequency $\mathbf{n}(\%)$ \\
\hline Mass media & $23(13.9)$ \\
Friends & $15(9.1)$ \\
Relatives & $12(7.3)$ \\
Public health midwife & $68(41.2)$ \\
General practitioner/ Gynaecologist & $16 \quad(9.7)$ \\
Missing data & $31(18.8)$ \\
\hline
\end{tabular}




\section{Discussion}

The present study describes the awareness, knowledge, attitudes and practice regarding cervical smear screening in a selected cohort of women in Sri Lanka. According to the results, awareness and knowledge is markedly poor with several aspects of the cervical cancer screening programme needing urgent attention. The findings of this study are consistent with several other studies from LMIC (9-12).

The only available previous Sri Lankan study on the awareness of Pap smear was done in 2013 as a survey among ever-married (women who have been married at least once in their lives) women between the ages of 25 and 65 years attending a medical clinic at the National Hospital of Sri Lanka (13). Data has been obtained on socio-demographic characteristics, awareness/ knowledge and uptake status of cervical smear testing and the investigators have concluded that the knowledge regarding cervical smear testing was poor, Pap smear uptake was very low and the parity and contact with patients with cervical cancer correlated with uptake. They recommended to increase the uptake through various campaigns (13). Several other studies have concluded that although knowledge of cervical smear as a screening procedure for cervical cancer is high, uptake of screening is low $(10,12)$.

Ranabhat S et al., in Nepal, have concluded that effective health education programmes should aim increasing knowledge about cervical cancer and screening as well as changing the attitude towards the test in a positive manner (9). In turn, this can contribute to increase the uptake of the cervical smear programme. A Gabonese study in 2014 revealed a very low level of knowledge about cervical malignancy and its prevention strategies. According to this study from Gabon, women's negligence was identified in $50 \%$ of them as a reason for not undergoing Pap smear screening (11). All these countries with lack of baseline awareness and knowledge belong to LMIC. In our study, $60 \%$ of women were not concerned about developing a cervical carcinoma themselves and $52.7 \%$ of them have never undergone cervical smear screening.

Present study also showed that $52.7 \%$ of subjects have not been subjected to cervical smear screening even though they were eligible. Further, 36.4\% women were not aware about a health facility where cervical screening is being performed. These issues highlight the deficiencies in our screening and prevention programme for cervical cancer. However, these women may have been informed that the hospital and/ or clinic would contact them if test result was unfavourable. In our study, fear of vaginal examination/ embarrassment has been reported to be in $6.7 \%$ indicating the place of social stigma and taboos in the society might have resulted in this. A recent review highlighted the social and cultural barriers for the participation of women in cervical smear programmes in LMIC (14). Not surprisingly, public health midwives are the commonest source of information proving their contribution to the gynaecological health of women in addition to their enormous contribution to the maternal and child health in Sri Lanka (15).

\section{Conclusion}

Cervical malignancy screening programme in a middle-income country like Sri Lanka needs some baseline rearrangements and improvement. The importance of public health policies with regards to increasing women's awareness, knowledge and attitude seems to be of paramount importance.

\section{Limitations}

This study was carried out in a single tertiary care centre with a non-probability sampling method limiting external validity of the results. Smaller sample size is a limitation.

\section{Acknowledgments}

We would like to express our sincere gratitude to the women participated in this survey. No specific funding received.

\section{Conflicts of interest}

The authors declare that they have no competing interests. 


\section{References}

1. National cancer control programme. Cancer incidence data Sri Lanka. 2015 [cited 2020 Feb 25]. Available from: http://www.nccp.health.gov.lk/images/PDF_PUBLICATI ONS/Cancer_Incidence_Data_2009.pdf

2. Patabendige $\mathrm{M}$, Wijesinghe $\mathrm{RD}$, Wijesuriya MWAB, Hapuachchige C. Surgical management of cervical cancer in a resource-limited setting: One year of data from the National Cancer Institute, Sri Lanka. Int J Gynecol Obstet. 2021; 152(1): 78-81.

3. World Health Organization. Cervical cancer, human papillomavirus (HPV), and HPV vaccines: Key points for policy-makers and health professionals [Internet]. 2007 [cited 2020 Jan 15]. Available from: https://apps.who.int/iris/bitstream/handle/10665/69873/W HO_RHR_08.14_eng.pdf; sequence=1

4. Ferlay J, Soerjomataram I, Dikshit R, Eser S, Mathers C, Rebelo $\mathrm{M}$, et al. Cancer incidence and mortality worldwide: Sources, methods and major patterns in GLOBOCAN 2012. Int JCancer. 2015; 136(5): E359-86.

5. World Health Organization. Improving data for decision-making: a toolkit for cervical cancer prevention and control programmes [Internet]. Geneva: World Health Organization; 2018. Available from: https://apps.who.int/iris/handle/10665/279420

6. Chelmow D. Cervical cancer screening and prevention. Obstet Gynecol. 2016; 127(1): e1-20.

7. Vale DB, Teixeira JC, Bragança JF, Derchain S, Sarian LO, Zeferino LC. Elimination of cervical cancer in lowand middle-income countries: Inequality of access and fragile healthcare systems. Int J Gynecol Obstet. 2021; 152(1): 7-11.

8. Programme NCC, Health M of, Lanka S. Prevention and Early Detection of Common Gynaecological Cancers; Guideline for Comprehensive Physicians, Primary Care. Available from: https://www.nccp.health.gov.lk/pdf/ publications/guidelines/Gynecological_Cancers.pdf
9. Ranabhat S, Tiwari M, Dhungana G, Shrestha R. Association of knowledge, attitude and demographic variables with cervical pap smear practice in Nepal. Asian Pacific J Cancer Prev. 2014; 15(20): 8905-10.

10. Thippeveeranna C, Mohan SS, Singh LR, Singh NN. Knowledge, attitude and practice of the pap smear as a screening procedure among nurses in a tertiary hospital in North Eastern India. Asian Pacific J Cancer Prev. 2013; 14(2): 849-52.

11. Assoumou SZ, Mabika BM, Mbiguino AN, Mouallif M, Khattabi A, Ennaji MM. Awareness and knowledge regarding of cervical cancer, Pap smear screening and human papillomavirus infection in Gabonese women. BMC Womens Health. 2015; 15(1): 1-7.

12. Almobarak AO, Elbadawi AA, Elmadhoun WM, Elhoweris MH, Ahmed MH. Knowledge, attitudes and practices of sudanese women regarding the Pap smear test and cervical cancer. Asian Pacific J Cancer Prev. 2016; 17(2): 625-30.

13. Shivanthan MC, Arunakiri K, Wickramasinghe SI, Sumanasekera RDN, Jayasinghe S, Rajapakse S. Low uptake of Pap smear testing among medical clinic attendees in a tertiary care hospital in Sri Lanka. Int Health. 2014; 6(2): 138-43.

14. Liebermann EJ, VanDevanter N, Hammer MJ, Fu MR. Social and Cultural Barriers to Women's Participation in Pap Smear Screening Programs in Low- and MiddleIncome Latin American and Caribbean Countries: An Integrative Review. J Transcult Nurs. 2018; 29(6): 591-602.

15. Senanayake H, Goonewardene $\mathrm{M}$, Ranatunga A, Hattotuwa R, Amarasekera S, Amarasinghe I. Achieving millennium development goals 4 and 5 in Sri Lanka. BJOG An Int J Obstet Gynaecol. 2011; 118(Suppl. 2): 78-87. 